\title{
MULTI-SIGNAL COMPRESSED SENSING FOR POLARIMETRIC SAR TOMOGRAPHY
}

\author{
E. Aguilera, M. Nannini, A. Reigber \\ Microwaves and Radar Institute, German Aerospace Center (DLR) \\ P.O. Box 1116, 82230 Wessling, Germany \\ Tel.: +49 - 8153281820, E-mail: Esteban.Aguilera@dlr.de
}

\begin{abstract}
In recent years, three-dimensional imaging by means of SAR tomography has become a field of intensive research. In SAR tomography, the vertical reflectivity function for every azimuth-range pixel is usually recovered by processing data collected using a defined repeat pass acquisition geometry. The most common approach is to generate a synthetic aperture in the elevation direction through imaging from a large number of parallel tracks. This imaging technique is appealing, since it is very simple. However, it has the drawback that large temporal baselines, which is the case for space-borne platforms, can severely affect the reconstruction. In an attempt to reduce the number of parallel tracks, we propose a new tomographic focusing approach that trades number of SAR images for correlations between neighboring azimuth-range pixels and polarimetric channels. As a matter of fact, this can be done under the framework of Distributed Compressed Sensing (DCS), which stems from Compressed Sensing (CS) theory, thus also exploiting sparsity in our tomographic signal. In addition, we address the problem of measurements affected by additive as well as multiplicative speckle noise. Results demonstrating the potential of the DCS methodology will be validated by using fully polarimetric L-band data acquired by the E-SAR sensor of DLR.
\end{abstract}

Index Terms - SAR tomography, polarimetry, compressed sensing, distributed compressed sensing.

\section{INTRODUCTION}

\subsection{Compressed sensing}

Compressed Sensing (CS) proposes measuring a signal $f$ by collecting $m$ linear measurements of the form $b=A f+z$, where $A$ is a $m$ by $n$ sensing matrix with $m$ typically smaller than $n$ by several orders of magnitude and $z$ is a noise term. The theory asserts that if $f$ is approximately sparse in a specific basis $\Psi$, it is indeed possible to recover $f$, under suitable conditions on the matrix $A$, by $L_{1}$ minimization

$$
\min _{\widetilde{f}}\|\Psi \widetilde{f}\|_{1} \text { subject to }\|A \widetilde{f}-b\|_{2} \leq \varepsilon
$$

where $\varepsilon$ is an upper bound on the noise level [1], [2], [3], [4].

\subsection{Distributed compressed sensing}

Distributed Compressed Sensing (DCS) theory enables the joint recovery of multi-signal ensembles by exploiting intersignal correlations. It generalizes the concept of a signal being sparse in some basis to the concept of an ensemble of signals being jointly sparse. In this paper, we demonstrate how to apply a multiple measurement vector model that has been thoroughly studied and can be found in the literature [5], [6]. One of the crowning achievements of this model is that it allows us to reduce the number of measurements needed for reconstruction. When it comes to SAR tomography, this translates into reducing the number of 2-D SAR images.

\subsection{Compressed sensing for SAR tomography}

In [7], CS inversion techniques for SAR tomography have proven to be applicable. The contribution of this paper is to extend this concept and take advantage of the inter-signal correlations between neighboring azimuth-range pixels as well as between polarimetric channels by means of DCS.

\section{PROBLEM FORMULATION}

Given three 3-D complex reflectivity functions $g_{h h}(x, r, s)$, $g_{v v}(x, r, s)$, and $g_{h v}(x, r, s)$ (one per polarimetric channel) of a specific area; where $x, r$, and $s$ are the azimuth, range, and elevation coordinates, respectively, and taking a small discretized subset of the space domain, i.e. a window of size $\Delta x, \Delta r, \Delta s$, so that $1 \leq x \leq \Delta x, 1 \leq r \leq \Delta r$, and $1 \leq s \leq \Delta s$, is there a way to compress these reflectivity functions? Do they share any information? If so, we can use this knowledge to recover them by means of the DCS machinery. Throughout this paper, the reflectivity functions will be 
represented as an ensemble of $P=\Delta x \Delta r$ signals along $s$. That is, $g_{h h}(p, s), g_{v v}(p, s)$, and $g_{h v}(p, s)$ with $1 \leq p \leq P$. Each signal of size $\Delta s$ will be denoted with the corresponding column vectors $g_{h h p}, g_{v v p}$, and $g_{h v p}$. Additionally, the tomographic sensing operation (using parallel tracks) for the signals in the $x y$ channel, will be jointly expressed as

$$
B_{x y}=\widetilde{\Phi} G_{x y}+Z_{x y}
$$

where

$$
B_{x y}=\left[\begin{array}{c}
b_{x y 1} \\
b_{x y 2} \\
b_{x y 3} \\
\vdots \\
b_{x y P}
\end{array}\right]
$$

and

$$
\widetilde{\Phi} G_{x y}=\left[\begin{array}{ccccc}
\Phi_{1} & 0 & 0 & \cdots & 0 \\
0 & \Phi_{2} & 0 & \cdots & 0 \\
0 & 0 & \Phi_{3} & \cdots & 0 \\
\vdots & \vdots & \vdots & \ddots & \vdots \\
0 & 0 & 0 & \cdots & \Phi_{P}
\end{array}\right]\left[\begin{array}{c}
g_{x y 1} \\
g_{x y 2} \\
g_{x y 3} \\
\vdots \\
g_{x y P}
\end{array}\right]
$$

The matrices $\Phi_{p}$, with $1 \leq p \leq P$, are the so-called steering matrices, $b_{x y p}$ is a stack (column vector) of $m$ pixels taken from $m$ corregistered SAR images that are measurements of $g_{x y p}$, and $Z_{x y}$ is a noise term. Expressions for the different polarimetric channels can be found by replacing $x y$ with $h h$, $v v$, or $h v$, accordingly. Finally, the support of a vector $w$ is defined as $\operatorname{supp} w=\left\{j, w_{j} \neq 0\right\}$.

\section{COMMON SUPPORT REGULARIZATION}

In this method, we suppose that all $P$ signals throughout polarimetric channels share, approximately, the same sparse support in the space domain but have different nonzero coefficients. This makes sense, as we are expecting backscatter from the same structure [8]. From (1), it follows that

$$
\left[\begin{array}{c}
B_{h h} \\
B_{v v} \\
B_{h v}
\end{array}\right]=\left[\begin{array}{ccc}
\widetilde{\Phi} & 0 & 0 \\
0 & \widetilde{\Phi} & 0 \\
0 & 0 & \widetilde{\Phi}
\end{array}\right]\left[\begin{array}{c}
G_{h h} \\
G_{v v} \\
G_{h v}
\end{array}\right]+\left[\begin{array}{c}
Z_{h h} \\
Z_{v v} \\
Z_{h v}
\end{array}\right],
$$

or $B_{\text {all }}=\widetilde{\Phi}_{\text {all }} G_{\text {all }}+Z_{\text {all }}$. And so, we can focus in all channels simultaneously by mixed $L_{2,1}$ minimization as follows

$$
\min _{\widetilde{G}}\|\widetilde{G}\|_{2,1} \text { subject to }\left\|\widetilde{\Phi}_{\text {all }} G_{\text {all }}-B_{\text {all }}\right\|_{F} \leq \varepsilon
$$

where $\|\bullet\|_{F}$ is the Frobenius matrix norm, $\|\bullet\|_{2,1}$ is the mixed norm (sum of the $L_{2}$ norms of the rows of a matrix), and $\widetilde{G}$ is constructed by concatenating the signals (column vectors) side by side as follows

$$
\widetilde{G}=\left[\begin{array}{lll}
\widetilde{G}_{h h} & \widetilde{G}_{v v} & \widetilde{G}_{h v}
\end{array}\right]
$$

where

$$
\begin{aligned}
\widetilde{G}_{h h} & =\left[\begin{array}{llll}
\widetilde{g}_{h h 1} & \widetilde{g}_{h h 2} & \cdots & \widetilde{g}_{h h P}
\end{array}\right], \\
\widetilde{G}_{v v} & =\left[\begin{array}{llll}
\widetilde{g}_{v v 1} & \widetilde{g}_{v v 2} & \cdots & \widetilde{g}_{v v P}
\end{array}\right], \\
\widetilde{G}_{h v} & =\left[\begin{array}{llll}
\widetilde{g}_{h v 1} & \widetilde{g}_{h v 2} & \cdots & \widetilde{g}_{h v P}
\end{array}\right] .
\end{aligned}
$$

Intuitively, the $L_{2,1}$ norm promotes sparsity along rows, while minimizing the energy along columns. As a result, the solution will be an ensemble of signals with significant overlap, which allows for polarimetric analyses. As a matter of fact, the authors in [6] proved that the probability of recovery failure decays exponentially in the number of columns of $\widetilde{G}$. This improvement can be understood from the fact that a mixed norm regularization rules out many of the possible subspaces where our solution might lie, thereby reducing the degrees of freedom in the optimization.

In practice, we may want to rephase every element of $B_{\text {all }}$, so that all pixels have a flat earth phase component based on the distance to the center of the window of size $\Delta x, \Delta r$. Thus,

$$
\Phi=\Phi_{1}=\Phi_{2}=\Phi_{3}=\ldots=\Phi_{p},
$$

which not only makes computations easier but also provides more accurate results.

\section{DENOISING BY SVD}

One of the main challenges in compressed sensing problems is an appropriate choice of the parameter $\varepsilon$, the upper bound on the additive noise level. Furthermore, in the presence of targets corrupted by multiplicative noise (speckle), even an accurate $\varepsilon$, is not of much help. Of course, coherent averaging of, say, the $P$ signals $b_{x y p}$ would not appropriately address the speckle noise problem, since this will render the resulting phase random as well.

By (6) and (10), the tomographic sensing operation for all polarimetric channels can be expressed as

$$
\widetilde{B}=\Phi \widetilde{G}+\widetilde{Z}
$$

where $\widetilde{Z}$ is our noise term,

$$
\widetilde{B}=\left[\begin{array}{ccc}
\widetilde{B}_{h h} & \widetilde{B}_{v v} & \widetilde{B}_{h v}
\end{array}\right],
$$

and

$$
\begin{aligned}
\widetilde{B}_{h h} & =\left[\begin{array}{llll}
b_{h h 1} & b_{h h 2} & \cdots & b_{h h P}
\end{array}\right], \\
\widetilde{B}_{v v} & =\left[\begin{array}{llll}
b_{v v 1} & b_{v v 2} & \cdots & b_{v v P}
\end{array}\right], \\
\widetilde{B}_{h v} & =\left[\begin{array}{llll}
b_{h v 1} & b_{h v 2} & \cdots & b_{h v P}
\end{array}\right] .
\end{aligned}
$$

Then, instead of solving (5), we propose the following minimization that is $\varepsilon$ independent

$$
\min _{\widetilde{G}}\|\widetilde{G}\|_{2,1} \text { subject to } D(\widetilde{B})=\Phi \widetilde{G}
$$


where $D(\bullet)$ is a denoising operator which in turn applies $D^{\prime}(\bullet)$ to each polarimetric channel

$$
D(\widetilde{B})=\left[\begin{array}{lll}
D^{\prime}\left(\widetilde{B}_{h h}\right) & D^{\prime}\left(\widetilde{B}_{v v}\right) & D^{\prime}\left(\widetilde{B}_{h v}\right)
\end{array}\right] .
$$

Let,

$$
\widetilde{B}_{x y}=\sum_{i=1}^{r a n k} \sigma_{x y i} U_{x y i} \otimes V_{x y i}^{*}
$$

be the singular value decomposition of $\widetilde{B}_{x y}$. Then, $D^{\prime}\left(\widetilde{B}_{x y}\right)=$ $\sigma_{x y 1} U_{x y 1}$.

Note, that we could keep more left singular vectors. However, when the number of tracks is small, the rank of $\widetilde{B}_{x y}$ is small, too, and the first singular value provides enough information. In addition, as in this case $\varepsilon=0$, it seems reasonable to discard some singular values to compensate. Nevertheless, it is worth mentioning that this denoising operation results in a loss of resolution in azimuth and range.

Recently, it has come to our attention that a similar approach for source localization has been used for slightly different purposes [9].

\section{EXPERIMENTAL RESULTS}

In order to demonstrate the potential of the outlined approach, we used five 2-D SAR images in the Pauli basis obtained by processing fully polarimetric L-band data. This data was acquired by the E-SAR sensor of DLR during a campaign near Dornstetten, Germany, in 2006. All flights were performed at approximately the same altitude with horizontal baselines of about $20 \mathrm{~m}$. Specifically, the center frequency used was $1.3 \mathrm{GHz}$, the nominal altitude above ground was about $3200 \mathrm{~m}$, with an azimuth and range resolution of $0.5 \mathrm{~m}$ and $1.5 \mathrm{~m}$, respectively.

In particular, we focused tomograms using the methodologies previously described at a fixed range distance of $4925 \mathrm{~m}$ and for 200 contiguous azimuth positions. As a result, we obtained tomograms in the azimuth and elevation directions of dimensions $200 \mathrm{~m}$ by $40 \mathrm{~m}$, respectively. The targets of interest are two corner reflectors which are in layover in every 2-D SAR image, and some grass, which is expected to be affected by speckle. Details on this layover geometry can be found in [10]. Lastly, the software we used to solve the minimization problems was CVXOPT [11].

In Fig. 1 (a) and (b), we took a 3 by 3 azimuth-range window and processed the tomograms individually for every azimuth-range position and for each polarimetric channel. For instance, for the $h h$ channel we solved

$$
\min _{\widetilde{g}_{h h p}}\left\|\widetilde{g}_{h h p}\right\|_{1} \text { subject to }\left\|\Phi_{p} \widetilde{g}_{h h p}-b_{h h p}\right\|_{2} \leq \varepsilon
$$

for every $p$ such that $1 \leq p \leq 9$. We will call this SSA (Single-Signal Approach), so as to distinguish it from the MSA (Multi-Signal Approach) as defined by (5) and (16). In
Fig. 1 (a), the average intensities are displayed per polarimetric channel ( $\mathrm{R}: \mathrm{HH}-\mathrm{VV}, \mathrm{G}: \mathrm{HV}, \mathrm{B}: \mathrm{HH}+\mathrm{VV})$. In Fig. 1 (b), we added the resulting average intensities together at a specific elevation for all polarimetric channels. In Fig. 1 (c) and (d), we took again a 3 by 3 azimuth-range window but did the processing according to (5) for all azimuth-range positions and polarimetric channels. Clearly, in Fig. 1 (c) and (d) the two corner reflectors can be easily be told apart. Additionally, it is easy to notice that in Fig. 1 (c) the colors are aligned, whereas in Fig. 1 (a) they are not.

Fig. 2 (a) and (b) correspond to Fig. 1 (b) and (d), respectively, but have been saturated to reveal possible artifacts. Evidently, in Fig. 2 (b) far fewer spurious spikes can be observed.

Fig. 3 presents normalized elevation profiles (span) for the exact azimuth-range position where the two corner reflectors lie. In Fig. 3 (a), we can see the profile found by Capon's method using ten passes and a 11 by 11 window. Fig. 3 (b) shows the average results obtained by processing the signals individually according to (19) using five passes and a 3 by 3 window. Finally, Fig. 3 (c) and (d) display the jointly processed average profile. Fig. 3 (c) uses the SVD denoising step and clearly exhibits more accuracy in the intensity. On the other hand, the intensities in Fig. 3 (d) seem to be a little affected, probably by an incorrect choice of $\varepsilon$.

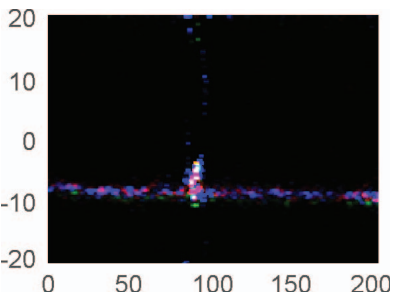

(a)

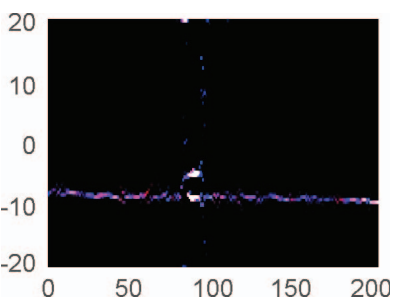

(c)

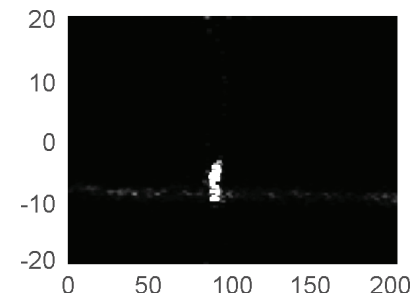

(b)

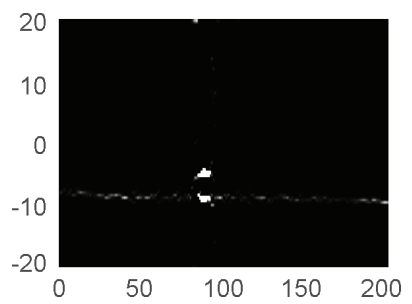

(d)
Fig. 1. Comparison between SSA and MSA (200 m by $40 \mathrm{~m}$ ). Two corner reflectors in layover and some grass: (a) Average intensities per polarimetric channel for SSA; (b) Average span for SSA; (c) Average intensities per polarimetric channel for MSA; (d) Average span for MSA.

\section{CONCLUSIONS}

A Distributed Compressed Sensing approach for polarimetric SAR tomography makes it possible to significantly reduce the 


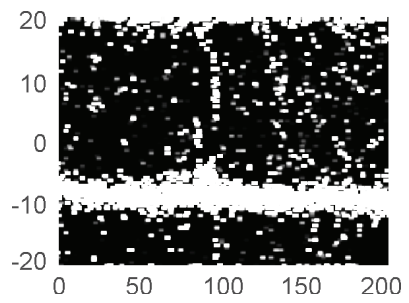

(a)

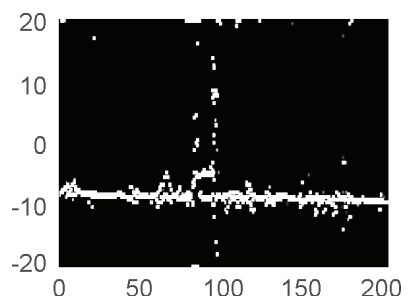

(b)
Fig. 2. Saturated average span (200 m by $40 \mathrm{~m}$ ) for (a) SSA and (b) MSA.

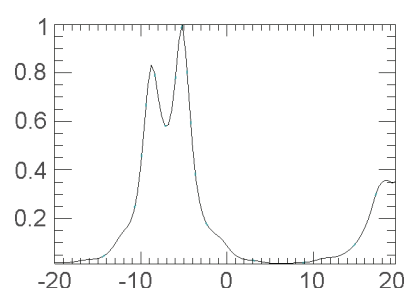

(a)

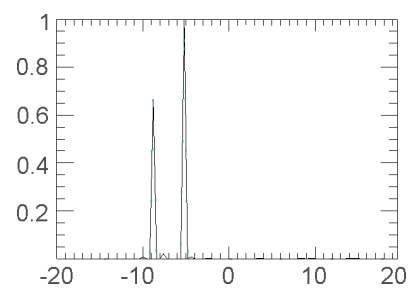

(c)

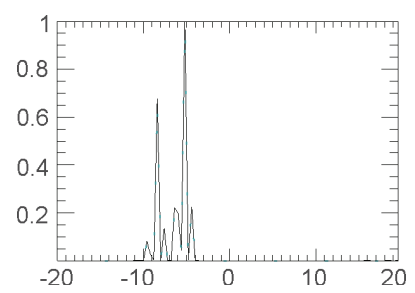

(b)

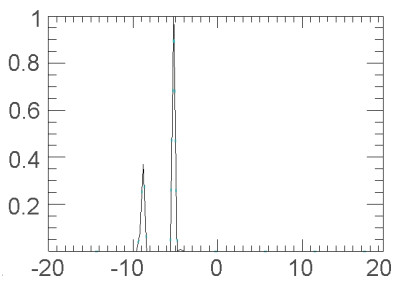

(d)
Fig. 3. Elevation profiles (normalized intensity) of two corner reflectors in layover obtained by: (a) the Capon's method; (b) SSA; (c) MSA with SVD denoising; (d) MSA.

number of required 2-D SAR images. In effect, even though the elevation profiles for each azimuth-range pixel are separately encoded, joint recovery of ensembles of polarimetric reflectivity functions allows exploiting their shared information.

In addition, the methods outlined allow for a robust polarimetric analysis of sparse solutions. Further, exploitation of multiple signals enables us to reduce spurious spikes or artifacts, which becomes even more accurate when denoising measurements previous to solving the minimization problem. Also, the focusing of deterministic as well as distributed targets shows a substantial improvement.

In the same spirit, a direct benefit of reducing the number of required passes is the possibility of studying the anisotropic behavior of scatterers in the elevation direction, since high resolution can be achieved using many small overlapping subapertures.

Future work will focus on including additional regularizations for targets that might not be sparse in the space domain, such as forests. As a matter of fact, elevation profiles are still extremely simple as compared with the behavior of the reflec- tivity function along azimuth and range. Hence, we are likely to find sparsifying bases, which may allow for analysis in the presence of volumetric scattering.

Finally, we would like to emphasize that the outlined SVD denoising step opens the door to a more robust analysis of speckled measurements, which can lead to sparse expansions in more sophisticated bases.

\section{REFERENCES}

[1] E. Candès, "Compressive sampling," in Proc. Int. Congr. Math., Madrid, Spain, vol. 3, pp. 1433-1452, 2006.

[2] R. Baraniuk, "Compressive sensing," IEEE Signal Process. Mag., vol. 24, pp. 118-121, Jul. 2007.

[3] D. Donoho, "Compressed sensing," IEEE Trans. Inf. Theory, vol. 52, pp. 1289-1306, Apr. 2006.

[4] E. Candès, J. Romberg, and T. Tao, "Robust uncertainty principles: Exact signal reconstruction from highly incomplete frequency information," IEEE Trans. Inf. Theory, vol. 52, pp. 489-509, Feb. 2006.

[5] D. Baron, M.F. Duarte, M.B. Waking, S. Sarvotham, and R.G. Baraniuk, "Distributed Compressive Sensing," Preprint, 2009.

[6] Y.C. Eldar and H. Rauhut, "Average Case Analysis of Multichannel Sparse Recovery Using Convex Relaxation," IEEE Trans. Inf. Theory, vol. 56, pp. 505-519, Jan. 2010.

[7] X.X. Zhu and R. Bamler, "Tomographic SAR Inversion by L1-Norm Regularization - The Compressive Sensing Approach," IEEE Trans. Geosci. Remote Sens., vol. 48, pp. 3839-3846, Oct. 2010.

[8] S. Tebaldini, "Algebraic Synthesis of Forest Scenarios From Multibaseline PolInSAR Data," IEEE Trans. Geosci. Remote Sens., vol. 47, pp. 4132-4142, Dec. 2009.

[9] D. Malioutov, M. Çetin, and A.S. Willsky, "A Sparse Signal Reconstruction Perspective for Source Localization With Sensor Arrays," IEEE Signal Process., vol. 53, pp. 3010-3022, Aug. 2005.

[10] M. Nannini, R. Scheiber, and A.Moreira, "Estimation of the minimum number of tracks for SAR tomography," IEEE Trans. Geosci. Remote Sens., vol. 47, pp. 531543, Feb. 2009.

[11] J. Dahl and L. Vandenberghe, "CVXOPT: Free software package for convex optimization," http://abel. ee.ucla.edu/cvxopt/. 